\title{
Tipologías de estudiantes de Fisioterapia según el uso que hacen del campus virtual
}

\author{
Physiotherapy undergraduates' typologies according to their use of \\ virtual campus
}

\author{
Mª Pilar Álvarez Vázquez*, Ana Álvarez-Méndez**, Carmen Bravo-Llatas***, \\ Jesús Cristóbal Barrios****, $\mathbf{M}^{\mathrm{a}}$ Teresa Angulo Carrere** \\ *Sección departamental de Biología Celular, Facultad de Medicina \\ **Departamento de Enfermería, Facultad de Enfermería, Fisioterapia y Podología \\ ***Área de Gobierno de Tecnologías de la Información y de Apoyo Técnico al Usuario \\ ****Área de Software Corporativo \\ Universidad Complutense de Madrid \\ 28040 Madrid, España \\ pilar@med.ucm.es amalvare@ucm.es mcbravo@ucm.es \\ jcristobal@ucm.es anguloca@enf.ucm.es
}

\begin{abstract}
Resumen
Presentamos en este artículo los resultados obtenidos tras procesar los registros del espacio virtual creado en la plataforma Moodle para la asignatura de "Anatomía Humana III", del grado en Fisioterapia. El análisis se realizó mediante el software libre para computación estadística y gráficos RStudio, un entorno de desarrollo integrado para el lenguaje de programación R. Un total de 19.611 registros correspondientes a la actividad realizada en el curso académico 2017/18 fueron depurados y anonimizados y posteriormente analizados. Las variables cuantitativas elegidas fueron el número total de visitas por día al curso virtualizado, el promedio de la frecuencia de visitas a la asignatura virtualizada por día de la semana y hora del día y por meses, el número de accesos a recursos, autoevaluaciones y a URLs. Además, se realizó el análisis estadístico de los datos con el software IBM SPSS v.25, comparando el uso del campus virtual con el rendimiento académico. Se realizaron pruebas de correlación de Spearman no paramétricas y árboles de decisión con dos criterios de corte. Los resultados obtenidos mostraron que el rendimiento académico de los estudiantes de esta asignatura está influido por el uso que hacen del campus virtual. Así, se ha visto que los alumnos con calificación inferior a 5 sobre 10 presentaron menor actividad en la plataforma Moodle, en todas las variables analizadas. Por el contrario, los estudiantes con calificaciones entre 8 y 10 sobre 10 mostraron una actividad en el espacio virtual significativamente mayor, especialmente en el número de visitas y en los recursos utilizados.
\end{abstract}

Palabras clave: Educación superior, Analítica del aprendizaje, Plataforma Moodle, Fisioterapia, Rendimiento académico, Tipologías de estudiantes de Grado.

\begin{abstract}
In this article we present the results obtained after processing the logs of the virtual space created for the subject "Human Anatomy III" (Physiotherapy Degree) in the Moodle platform. The analysis was performed using free software for statistical computing and RStudio graphics, an integrated development environment for R. A total of 19,611 logs, corresponding to the activity recorded in the 2017-2018 academic year were extracted, debugged and anonymized, to be analysed. The quantitative variables analysed were: the total number of visits to the virtualized course, the average of visits by weekday, by hours of the day and along the quarter, as well as the number of accesses to resources, self-assessments and URLs. In addition, the statistical analysis of the data was performed with the IBM
\end{abstract}

Los autores agradecen la cooperación de los estudiantes y el apoyo recibido desde el Vicerrectorado de Tecnologías de la Información y la Oficina eCampus de la Universidad Complutense de Madrid.

Parte de los resultados de este trabajo fueron presentados en el XXIV Congreso de la SEDEM, Salamanca 6-8 noviembre 2019. 
SPSS v.25 software, analysing the relationship between the use of the virtual campus and academic performance. Non-parametric Spearman correlation tests and decision trees with two cut criteria were performed. The results obtained showed that the academic performance of the students of this subject is determined by their use of the virtual campus. Thus, it has been observed that students who failed (grades below 5 out of 10) had less activity on the Moodle platform, in all the variables analysed. By contrast, students with higher marks (grades between 8 and 10 out of 10) showed a significantly higher activity in the virtual space, especially in the number of visits and in the resources used.

Key words: Higher Education, Learning Analytics, Moodle platform, Physiotherapy, Academic performance, Undergraduates' typologies.

\section{Introducción}

El uso de las nuevas Tecnologías de la Información y la Comunicación (TIC) continúa revolucionando el mundo de la educación a través del cambio de las formas y técnicas de impartir docencia. Las universidades y las instituciones de educación superior utilizan diferentes plataformas que proporcionan entornos digitales de trabajo con acceso privado. De entre ellas, la plataforma Moodle es una de las más utilizadas en el campo docente universitario, su uso a través de internet permite el diseño de herramientas que complementan, estimulan y mejoran el aprendizaje del estudiante (Konstantinidis y Grafton, 2013). Este espacio digital, que simula espacios físicos para la enseñanza y el aprendizaje, facilita la interacción entre los participantes, además de permitir la monitorización de los usuarios (Chaparro et al., 2010; Jenaro et al., 2018; Torres-Porras et al., 2018). Las diferentes interacciones realizadas por los estudiantes quedan almacenadas en la plataforma virtual, de forma que esos datos se pueden analizar revelando comportamientos y preferencias (Yu y Jo, 2014; Shahiri et al., 2015).

Las actividades de los estudiantes en las plataformas virtuales generan una enorme cantidad de datos en forma de registros o logs. Sin embargo, todos esos datos se almacenan de forma desestructurada y sin sentido por lo cual los docentes tendrían grandes dificultades para extraer información relevante y conclusiones (Konstantinidis y Grafton, 2013). El uso de técnicas de Learning Management Systems (LMS) permite analizar grandes cantidades de datos, de manera automática o semiautomática, abriendo un nuevo campo de investigación para docentes, al ser posible identificar patrones repetitivos, tendencias o reglas que expliquen el comportamiento de los datos en un determinado contexto (Brown 2011, Sin y Muthu 2015; Domínguez et al. 2016; Rossetti et al., 2017). Como afirman Konstantinidis y Grafton (2013), los LMS nos permiten agregar contexto a los números, correlacionar diferentes conjuntos de datos y hacer visible lo invisible.

El rendimiento académico de los estudiantes es una parte esencial en las instituciones de educación superior, no sólo por el beneficio de los propios estudiantes sino porque, además, el historial de logros académicos constituye uno de los criterios de la alta calidad y excelencia universitaria (Shahiri et al., 2015). Varios autores han destacado la importancia de analizar la actividad E-learning, con la finalidad de evaluar su efectividad sobre el rendimiento académico de los estudiantes (Macfadyen y Dawson, 2010; Kotsiantis et al., 2013; Mwalumbwe y Mtebe, 2017). Estudios recientes demuestran la existencia de diferentes patrones en el comportamiento de los estudiantes en el uso de la plataforma virtual (Chaparro et al., 2010; Lee et al., 2016; Torres-Porras et al. 2018). No sólo parece existir un comportamiento común en algunos grupos que obtienen rendimientos académicos similares (Jenaro et al., 2018) sino que, además, existen variables controlables que pueden llegar a predecir los resultados de aprendizaje de manera más significativa (Yu y Jo 2014).

\section{Contexto y objetivos}

Este estudio es parte del proyecto de innovación y mejora de la calidad docente titulado "Lo que no conocemos de nuestros estudiantes de CC de la Salud y nos revelan los rastros digitales. Learning 
Analytics: herramienta con potencial transformador para mejorar nuestra docencia" concedido por el Vicerrectorado de Calidad de la Universidad Complutense de Madrid en el curso 2017/18. En él se analizaron los registros almacenados en Moodle correspondientes a nueve espacios y asignaturas de tres grados (Medicina, Fisioterapia y Podología), con el fin de conocer los hábitos de nuestros estudiantes en el campus virtual (CV), determinar si existen distintas tipologías de estudiantes de acuerdo al uso que hacen del mismo y, en su caso, establecer si presentan diferencias en cuanto a su rendimiento académico.

En este trabajo presentamos los resultados del análisis de la asignatura de Anatomía Humana III, de carácter básico para el Grado en Fisioterapia (UCM, 2019). Se imparte en el primer cuatrimestre en segundo curso y su contenido se centra en la biomecánica del aparato locomotor. Presenta una carga lectiva de 6 créditos ECTS distribuidos en clases magistrales, impartidas 3 días en semana, y sesiones de prácticas organizadas a lo largo del cuatrimestre, todas ellas en horario de mañana. Se evalúan los comportamientos de los estudiantes de acuerdo al número de visitas registradas, la frecuencia de uso, el mapa cronológico y las actividades realizadas. Así mismo, se analiza si las distintas tipologías de estudiantes muestran diferencias en el rendimiento académico.

Los estudiantes de la asignatura de Anatomía Humana III del Grado en Fisioterapia son personas con un alto grado vocacional y potencial académico, con buenos rendimientos y aprendizajes. El objetivo de este trabajo es analizar su comportamiento en el espacio virtual creado para dar soporte a esta asignatura (en adelante, "asignatura virtualizada"), describir si existen distintas tipologías de estudiantes, y la posible relación entre el uso que hacen del CV y el rendimiento académico.

\section{Metodología}

La profesora encargada de Anatomía Humana III en el curso 2017/18 diseñó un aula virtual en el CV como apoyo a la enseñanza, con distintos tipos de recursos como son la guía docente, el programa de la asignatura, los criterios de evaluación, los horarios de tutorías, presentaciones de clase, enlaces a recursos externos (URLs), 4 cuestionarios y un foro de avisos. Los enlaces a URLs son vínculos a vídeos propios (Angulo y Álvarez-Méndez, 2007) elaborados en un proyecto de innovación educativa anterior, destinados principalmente al aprendizaje práctico. Los cuestionarios son autoevaluaciones voluntarias y evaluables que se abren en fechas concretas para que el estudiante pueda entrenar y evaluar el aprendizaje de los contenidos de cara al examen final de teoría.

Al término del curso, se extrajeron los registros de la asignatura virtualizada almacenados en Moodle. Un total de 19.611 registros fueron depurados, anonimizados, procesados y estandarizados. En este procesamiento se eliminaron los que no correspondieran a participantes con rol de estudiantes. Seguidamente, los registros fueron analizados con R, un lenguaje de programación con enfoque al análisis estadístico y la representación gráfica, y con RStudio, un entorno de desarrollo integrado para el lenguaje de programación R. El programa R permite visualizar los resultados en distintos tipos de gráficos.

Para la comparativa de la asignatura se procesaron los registros guardados en Moodle correspondientes a la titulación del Grado en Fisioterapia, los cuales fueron proporcionados por el Vicerrectorado de Tecnologías de la Información.

Adicionalmente, los datos agregados fueron analizados con el programa IBM SPSS v25. El análisis estadístico realizado incluyó correlaciones paramétricas y no paramétricas de Spearman, así como árboles de decisión mediante dos criterios de corte (CHAID: minimización de la significatividad del estadístico de Fisher-Snedecor, y CRT: minimización de la varianza ponderada de los nodos hijos) para relacionar rendimiento académico con segmentos de estudiantes con una actividad similar en CV. 


\section{Resultados}

En el curso 2017/18 se matricularon en Anatomía Humana III 69 estudiantes, siendo el 65\% mujeres. El espacio digital fue muy frecuentado puesto que el $100 \%$ de los estudiantes lo visitó, accedió a recursos y realizó cuestionarios, el $96,67 \%$ accedió a $U R L s$, si bien solo el $20 \%$ consultó en los foros.

La actividad analizada mostró una mayor frecuencia los cuatro primeros días de la semana, especialmente el lunes, coincidiendo con los días de impartición de clases de la asignatura. En cuanto al horario, la mayor actividad de visitas se produjo a partir de las 15:30 horas, cuando finalizaban las clases teóricas y talleres prácticos (Figura 1).

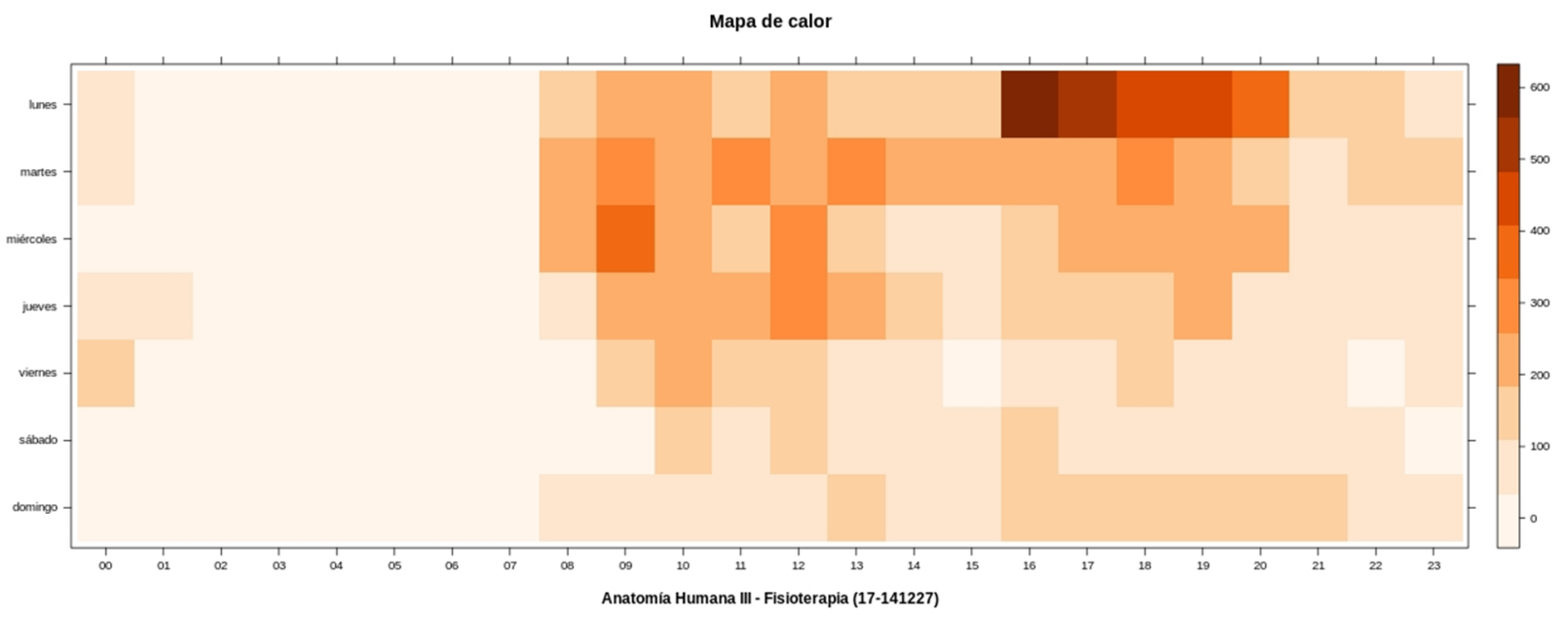

Figura 1: Mapa de calor de las visitas al CV de Anatomía Humana III (17-141227) según días de la semana y horas del día. Los colores más oscuros representan mayor número de visitas

Cuando se comparó la actividad de los estudiantes de Anatomía Humana III con la actividad media registrada en el conjunto de asignaturas del Grado en Fisioterapia se encontró que fue superior cada día de la semana, como se observa en la Figura 2.

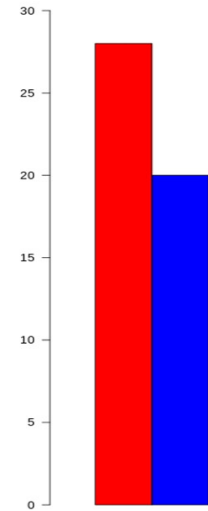

Iun

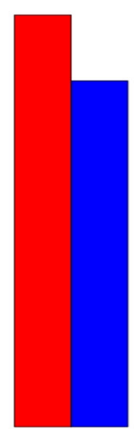

mar

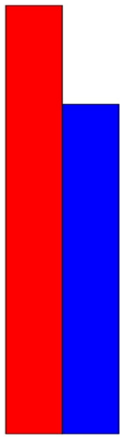

mié

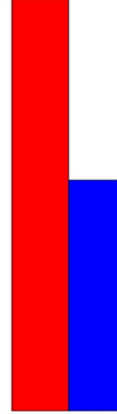

jue

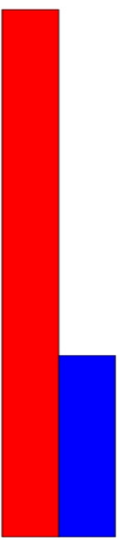

vie

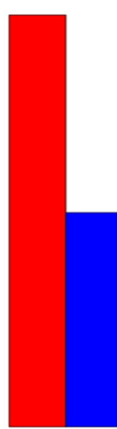

sab

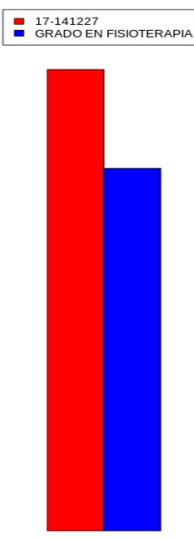

dom

Figura 2: Frecuencia de uso diario del CV de Anatomía Humana III (17-141227) comparada con la media de uso del total de asignaturas del grado en Fisioterapia 
El análisis de la actividad registrada mostró una estrecha relación con el calendario académico. Como se aprecia en la Figura 3, tanto el número de visitas como de consultas a recursos, a URLs y a cuestionarios estuvo determinado claramente por las fechas de los exámenes (líneas rojas) y de las autoevaluaciones (líneas grises).
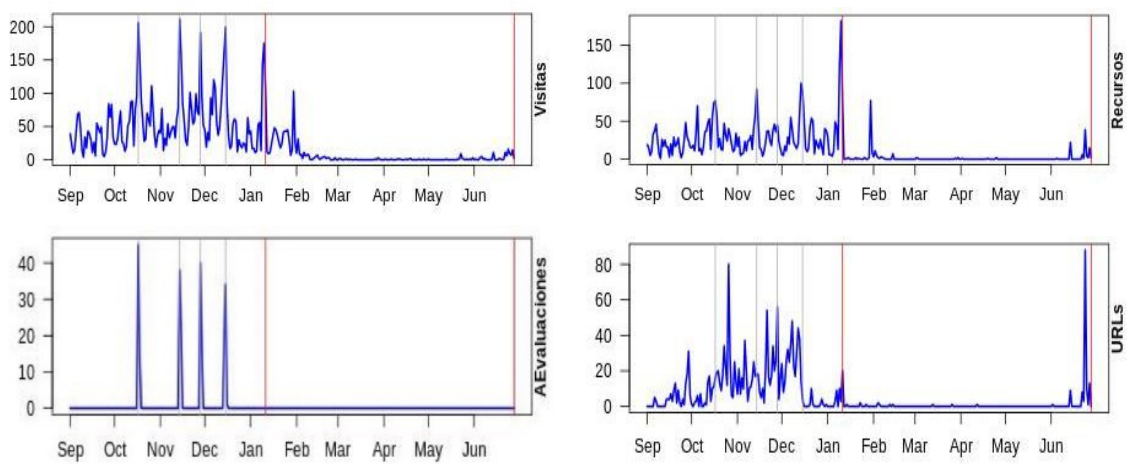

Figura 3: Actividad registrada en el CV de Anatomía Humana III (17-141227) (frecuencia de accesos, descargas de recursos y enlaces a URLs, autoevaluaciones)

Al cruzar los registros analizados con las calificaciones finales obtenidas por los estudiantes, el programa $\mathrm{R}$ elaboró gráficos de estrella multivariable de Kiwiat en los que representa el comportamiento según segmentos de calificaciones. Los resultados indicaron que cada cluster de estudiantes, de acuerdo a su nota final, se caracterizó por un uso diferencial del CV. Así, en la Figura 4 se aprecia que las medias de visitas, accesos a recursos, a URLs y a cuestionarios es distinto en cada cluster.

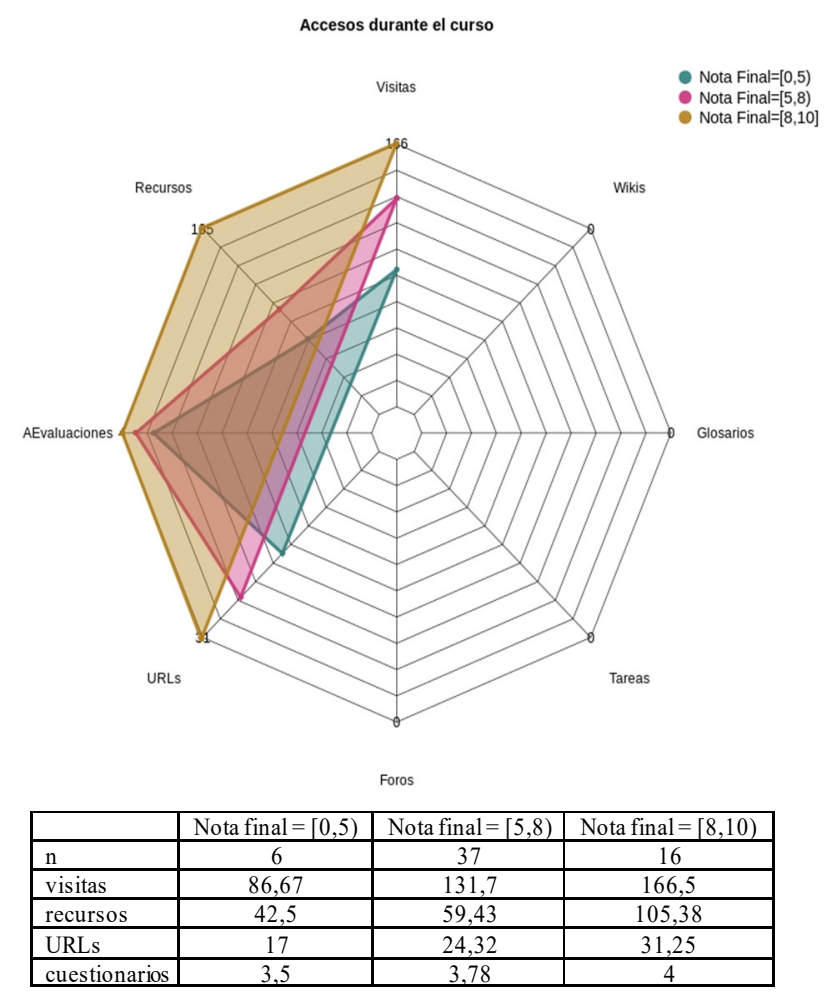

Figura 4: Clusters de estudiantes según su calificación final y actividad en el CV de Anatomía Humana III (17-141227). Los datos indican los valores medios de los estudiantes que integran cada cluster 
El análisis estadístico reveló algunas correlaciones no paramétricas con el test Rho de Spearman. Así, la nota de prácticas correlacionó significativamente con el acceso a $U R L s(\mathrm{r}=0,283 \mathrm{p}<0,05)$ en tanto que la nota de teoría correlacionó significativamente con visitas $(\mathrm{r}=0,282 \mathrm{p}<0,01)$ y acceso a recursos $(\mathrm{r}=0,372$ $\mathrm{p}<0,01)$. Igualmente, la calificación final en primera convocatoria se correlacionó con visitas $(\mathrm{r}=0,302 \mathrm{p}<$ $0,05)$ y acceso a recursos $(r=0,396 \mathrm{p}<0,01)$.

Cuando se ha estudiado la existencia de clusters mediante árboles de decisión, se ha encontrado que la calificación obtenida en primera convocatoria estuvo claramente influida por el número de cuestionarios realizados y de accesos a recursos, como se observa en la Figura 5.

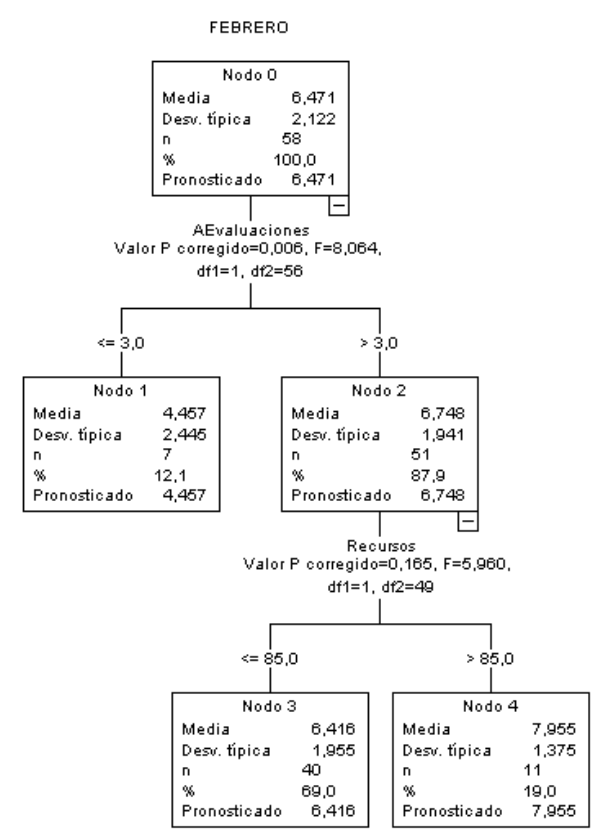

Figura 5: Árbol de decisión según calificación en primera convocatoria (método de crecimiento CHAID, calidad estimada-varianza ponderada intranodos: 3,514 )

Asimismo, los árboles de decisión obtenidos para las notas finales de teoría y de prácticas (Figura 6) revelaron que la realización de los cuestionarios son determinantes para la nota final del examen teórico, mientras que la variable que influye en las notas del examen práctico, es el acceso a URLs.
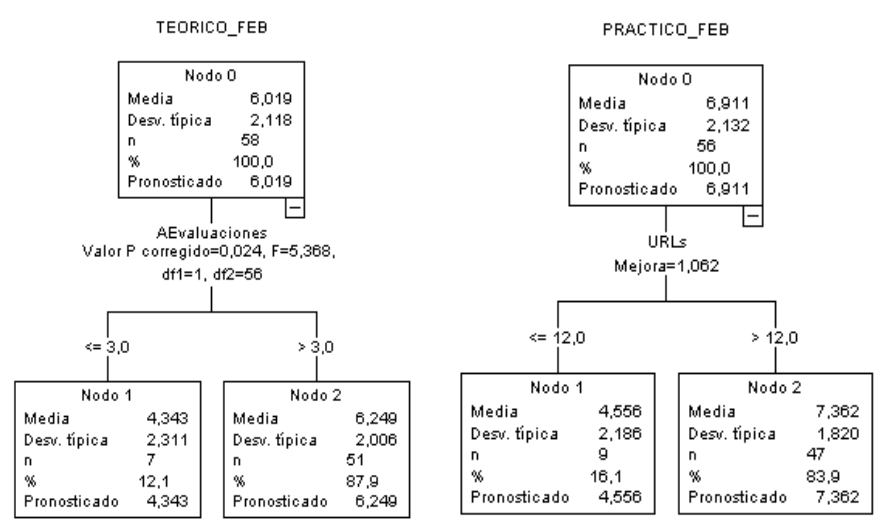

Figura 6: Árboles de decisión según nota de teoría (método de crecimiento CHAID, calidad estimada 4,023) y de prácticas (método de crecimiento CRT, calidad estimada 3,403) en primera convocatoria.

Calidad estimada: varianza ponderada intranodos 


\section{Discusión y conclusiones}

En este artículo presentamos datos objetivos que analizan la relación entre el proceso de enseñanza aprendizaje, el uso de TIC y el rendimiento académico. En las asignaturas presenciales, el uso de plataformas facilita no solo el intercambio de información en todo tipo de formatos y el seguimiento de los alumnos, sino que cada uno ajuste su ritmo de aprendizaje (Jenaro et al. 2018). Los registros analizados muestran que las visitas a la asignatura virtualizada y los accesos a los recursos y herramientas facilitadas fueron constantes a lo largo del cuatrimestre, con tasas mayores que la media de la titulación, y con una clara dependencia de las fechas clave en la evaluación, lo que está en consonancia con resultados obtenidos en otros espacios analizados dentro del proyecto (Álvarez et al. 2020).

A pesar de que los porcentajes de éxito en Anatomía Humana III son elevados y la implicación de los alumnos es alta, es posible encontrar diferentes tipologías de estudiantes, siendo los que muestran un mayor uso del CV los que logran mejores calificaciones, y al revés, los estudiantes que usan menos el CV, obtienen peores notas. La correlación positiva entre el comportamiento en el CV y las notas se obtiene tanto para la parte teórica como para la práctica. Estas diferencias de comportamiento se revelan igualmente al elaborar árboles de decisión, lo que permite concluir que el aprovechamiento de los recursos y herramientas que se facilitan en el CV permite predecir un mejor rendimiento académico, tanto en las notas del examen de teoría como en el de prácticas y, por tanto, en la calificación final de la asignatura. Estos resultados están en línea con otros trabajos (Kotsiantis et al. 2013; Yu y Jo, 2014; Cerezo et al. 2016).

Para el profesorado es importante saber que, aunque con las TIC la carga de trabajo ha aumentado considerablemente, el esfuerzo y el tiempo dedicados a la elaboración y diseño de recursos y actividades redundan efectivamente en un mejor aprendizaje de los estudiantes. Así, el profesor sigue siendo determinante en el rendimiento académico y su papel como administrador del espacio digital resulta esencial. Consideramos importante transmitir a nuestros alumnos la importancia de hacer un buen uso del $\mathrm{CV}$ ya que hay evidencias claras de que las participaciones más activas se relacionan con mejores rendimientos.

\section{Referencias}

Álvarez, M.P., Álvarez-Méndez, A., Angulo, M.T., Cristóbal, J., Bravo-Llatas, M.C. (2020) Learning analytics in human histology reveals different student clusters and different academic performance. Proceedings of INTED 2020, 14th International Technology, Education and Development Conference, en prensa.

Angulo, M.T., Álvarez-Méndez, A. (2007) CD-ROM interactivo para valoración biomecánica de la extremidad inferior. III Jornada Campus Virtual UCM: Innovación en el Campus Virtual: metodologías y herramientas. Editorial Complutense, Madrid, pp. 299-300.

Brown, M. (2011) Learning Analytics: the coming third wave. EDUCAUSE Learning Initiative. https://ibrary.educause.edu/-/media/files/library/2011/4/elib1101-pdf.pdf

Cerezo, R., Sánchez-Santillán, M., Puerto, M., Núñez, J.C. (2016) Students' LMS intereaction patterns and their relationship with achievement: A case study in higher education. Computers \& Education, 96, pp. 42-54.

Chaparro, J., Iglesias, S., Pascual, F. (2010) Uso del registro de actividad de Moodle para un estudio del rendimiento académico de alumnos en entornos en línea y presencial. 4th International Conference on Industrial Engineering and Industrial Management XIV Congreso de Ingeniería de Organización, pp. 753-760. Donostia-San Sebastián.

Domínguez, D., Álvarez, J., Gil-Jaurena, I. (2016) Analítica del aprendizaje y Big Data: heurísticas y marcos interpretativos. DILEMATA, International Journal of Applied Ethics, 22, pp. 87-103.

Jenaro, C., Castaño, R., Martín, M.E., Flores, N. (2018) Rendimiento académico en educación superior y su asociación con la participación activa en la plataforma Moodle. Estudios Sobre Educación, 34, pp. 177-198. 
Kotsiantis, S., Telios, N., Filippidi, A., Komis, V. (2013) Using learning analytics to identify successful learners in a blended learning course. International Journal of Technology Enhanced Learning, 5(2), pp. 133-150.

Konstantinidis, A., Grafton, C. (2013) Using Excel Macros to Analyse Moodle Logs. Conference Proceedings. 2nd Moodle Research Conference, pp. 33-39. Sousse, Tunisia.

Lee, J.E., Recker, M.M., Choi, H., Hong, W.J., Kim, N.J., Lee, K., Lefler, M., Louviere, J., Walker, A. (2016) Applying Data Mining Methods to Understand User Interactions within Learning Management Systems: Approaches and Lessons Learned. Journal of Educational Technology Development \& Exchange, 8(2), pp. 99-116.

Macfadyen, L.P., Dawson, S. (2010) Mining LMS data to develop an "earlywarning system" for educators: A proof of concept. Computers \& Education, 54(2), pp. 588-599.

Mwalumbwe, I., Mtebe, J. (2017) Using Learning Analytics to predictt students performance in Moodle Learning Management System: A case of Meya University of Science and Technology. The Electronic Journal of Information Systems in Developing Countries (EJISDC), 79(1), pp. 1-13.

Rossetti, S.R., Verdugo, M.L., Bayliss, D. (2017) Learning Analitics para determinar la realción entre uso de un Learning Management System y rendimiento académico. XXII Congreso Internacional de Contaduría, Administración e Informática. Ciudad de Méjico.

Shahiri, A.M., Husain, W., Rashid, N.A. (2015) The Third Information Systems International Conference. Procedia Computer Science, 72, pp. 414-422.

Sin, K., Muthu, L. (2015) Application of Big Data in education data mining and Learning Analitics. A literature review. Ictact Journal on Soft Computing: Special Issue on Soft Computing Model and Big Data Models for Big Data, 5(4), pp. 1035-1049.

Torres-Porras, J., Alcántara, J., Rubio, S. (2018) Virtual platforms use: a useful monitoring tool. EDMETIC. Revista de Educación Mediática y TIC, 7(1), pp. 242-255.

UCM (2019) Guía docente de la asignatura Anatomía Humana III, Grado en Fisioterapia. Recuperado de: https://enfermeria.ucm.es/data/cont/media/www/pag-128986/Anatom\%C3\%ADa\%20Humana $\% 20$ III.pdf

Yu, T., Jo, I.H. (2014) Educational technology approach toward learning analytics: relationship between student online behavior and learning performance in higher education. Proceedings of the Fourth International Conference on Learning Analytics And Knowledge, pp. 216-271. 